\title{
The Channel Estimation and Modeling in High Altitude Platform Station Wireless Communication Dynamic Network
}

\author{
Xiaoyang Liu, ${ }^{1,2}$ Xiaoping Zeng, ${ }^{1}$ Chao Liu, ${ }^{2}$ Wanping Liu, ${ }^{2}$ and Yihao Zhang ${ }^{2}$ \\ ${ }^{1}$ Postdoctoral Research Station of Information and Communication Engineering, Chongqing University, Chongqing 400030, China \\ ${ }^{2}$ School of Computer Science and Engineering, Chongqing University of Technology, Chongqing 400054, China \\ Correspondence should be addressed to Xiaoyang Liu; 1xy3103@163.com
}

Received 6 February 2017; Revised 27 April 2017; Accepted 7 May 2017; Published 30 May 2017

Academic Editor: Filippo Cacace

Copyright (c) 2017 Xiaoyang Liu et al. This is an open access article distributed under the Creative Commons Attribution License, which permits unrestricted use, distribution, and reproduction in any medium, provided the original work is properly cited.

\begin{abstract}
In order to analyze the channel estimation performance of near space high altitude platform station (HAPS) in wireless communication system, the structure and formation of HAPS are studied in this paper. The traditional Least Squares (LS) channel estimation method and Singular Value Decomposition-Linear Minimum Mean-Squared (SVD-LMMS) channel estimation method are compared and investigated. A novel channel estimation method and model are proposed. The channel estimation performance of HAPS is studied deeply. The simulation and theoretical analysis results show that the performance of the proposed method is better than the traditional methods. The lower Bit Error Rate (BER) and higher Signal Noise Ratio (SNR) can be obtained by the proposed method compared with the LS and SVD-LMMS methods.
\end{abstract}

\section{Introduction}

1.1. Background and Research Status. High altitude platform station (HAPS) is a stranded in $20 \mathrm{~km} \sim 50 \mathrm{~km}$ height of near space, a particular location which is a stationary platform relative to the ground. It can be seen as between terrestrial communication system and satellite communication system between communications system $[1,2]$. It is aimed at the height of the development of land and space between spaces of potential interest. It can improve the communication capacity and spectrum in efficiency and also can reduce the equipment cost and complexity. The path decline of HAPS is smaller than terrestrial communication system, and they have smaller delay than the satellite communication system. In order to be more competitive, more and more people begin to pay attention to HAPS. In the near space platform, construction of monitoring and communication system received further attention. The development of HAPS is similar to satellite communication in the 1960s [3-5]. HAPS can be described as "a new wireless communication technology that has a broad application prospect and can overturn the development of telecom industry new technology." It is the foundation of the next generation of wireless communication. It has to make full use of radio spectrum resources, large capacity of the system user, communication of good quality, low operational risk, and so on. You can upgrade communication load operation at any time.

References $[6,7]$ present a suitable high-speed mobile near space HAPS channel estimation pilot sequence based on the traditional optimal wireless communication system pilot sequence. Each received-transmit antenna of HAPS wireless communication space system can be considered as independent HAPS system after insertion of the pilot sequence $[8,9]$. ICI (Intersymbol Interference) coefficient and DFT (Discrete Fourier Transform) channel estimation algorithm are researched in $[10,11]$; as long as this relationship is defined, we can get the outputs only by nonlinear computation. Furthermore, the feedback signal is calculated. It can use CAZAC (Constant Amplitude Zero Autocorrelation) sequence [12-15] as training sequence for channel estimation with fast moving system environment. This sequence will ensure the independence of each transmit antenna pilot frequency which can eliminate the interference of the pilot frequency when doing the channel estimation $[10,16,17]$. Transmitting antenna can send pilot frequency alternately which will avoid aliasing, namely, when transmitting pilot 
frequency information by one antenna while other antennas remain idle. Thus, channel response can be easily estimated between receiving-sending antennas. But in high-speed timevarying mobile environment, due to data subcarrier to pilot frequency subcarrier interference [11, 18], it is difficult to estimate each response to transmit-receive channel, thus seriously affecting the overall performance of the system. So it is said that the structure is only suitable for the slowly varying channel, and for high-speed mobile channel, this method cannot track channel changes [19-21].

1.2. Motivations. The above discussion implies that novel inequality methods allow us to obtain more precise SNR and lower BER. Most references do not consider the dynamic behaviors of wireless communication networks under near space. Wireless communication channel estimation modeling method inequality is presented in [22-25]. Under this method efficiency, novel channel estimation method and mathematics model could be proposed.

1.3. Our Work and Contributions. In this paper, the wireless communication channel estimation and modeling problem of near space by dynamic method is further investigated. By giving more precise estimation for the SNR and the lower BER, a new channel estimation method is obtained. Under this method, some novel results are presented. Based on the discrete dynamics topology structure, the fast dynamic channel mathematics model is derived. Simulation is employed to indicate the effectiveness of the proposed method and model.

The main contributions of this paper are listed as follows. (1) A new wireless communication framework is introduced to improve the channel performance. (2) A novel channel estimation method and a new channel model are proposed. (3) A new discrete dynamics topology structure in network is built up. (4) The lower BER and higher SNR are obtained by the theoretical simulation results. These results are practical and objective.

1.4. Structure of the Paper. The rest of this paper is organized as follows. In Section 2, HAPS wireless communication network is introduced. In Section 3, LS (Least Squares) channel estimation principle and SVD-LMMS's channel estimation principle are studied. In Section 4, a new channel estimation and modeling method is proposed. In Section 5, the simulation analytical results are presented. Finally, conclusions are drawn in Section 6.

\section{HAPS Network}

2.1. Structure and Formation of HAPS Network. Figure 1 shows the abstract model of the near space HAPS network system. HAPS complex network includes a set of forward that can perform routing and traffic management HAPS communication node. It can be implemented with a laser or microwave communication between them. It is a challenging problem about the link between HAPS [26].

The geometric relationship of communications system of HAPS is shown in Figure 2.

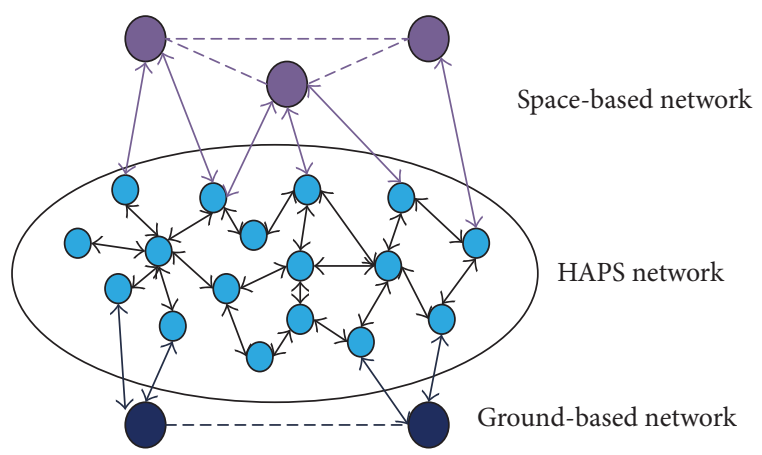

FIGURE 1: The abstract model of the near space HAPS network system.

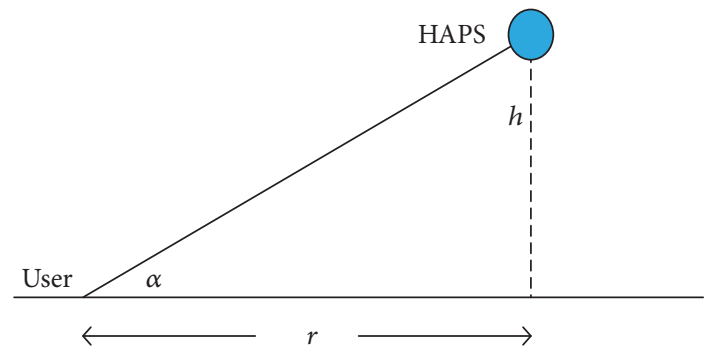

FIGURE 2: The geometric relationships of communications system.

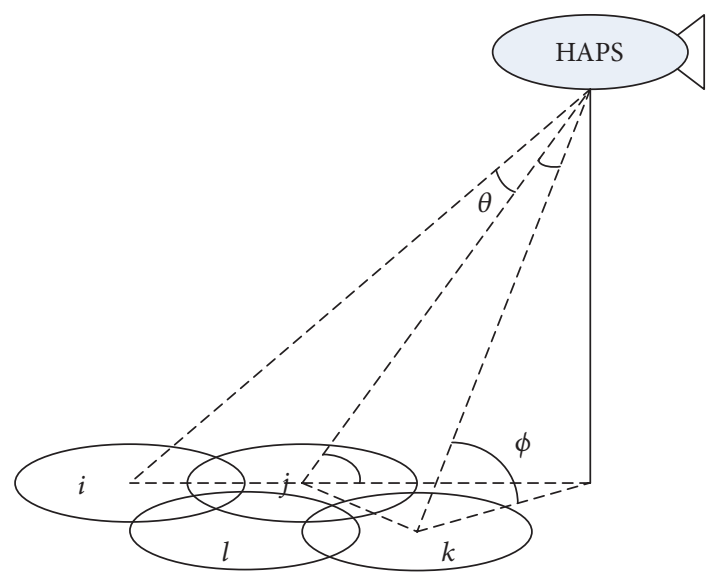

FIGURE 3: The communication network structure of HAPS.

Within the scope of the near-earth space, we use stable communications platform as a microwave relay station. With the ground control equipment, entrance equipment and a variety of wireless communication systems are in the composition of the user. High altitude platform can be comprehensive network and satellite ground and also can separate and ground network, as can be seen in Figure 3. The communication platform to keep in sync with the earth's rotation can reside in the air for a long time. High 


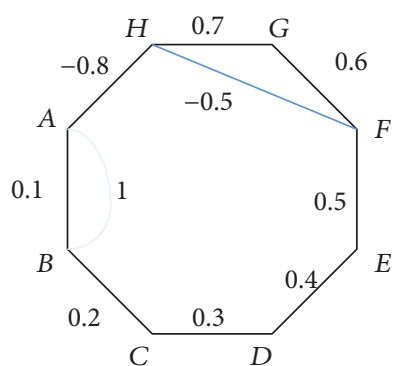

(a)

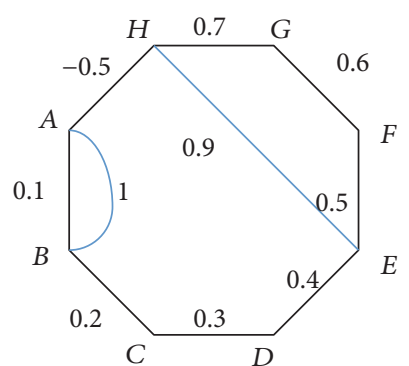

(b)

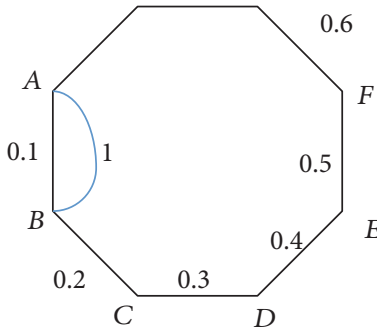

(c)

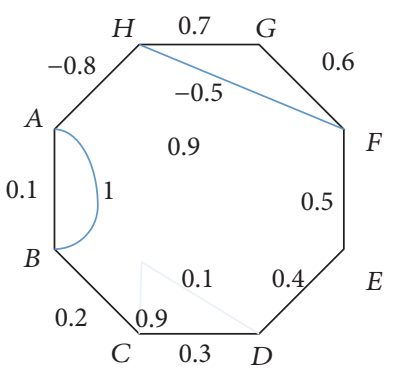

(d)

FIGURE 4: Network topology structure.

altitude platform communication has good waves transmission characteristics [27]. HAPS is a key instrumentality for broadband wireless communication, as it could complement and cooperate with satellite and terrestrial communications.

2.2. Discrete Dynamics Topology Structure in Network. Suppose that there are $n$ nodes in the HAPS network. The complex dynamical HAPS network can be described as follows $[28,29]$ :

$$
\mathbf{x}_{i}(k+1)=\mathbf{f}_{i}\left(\mathbf{X}_{i}(k)\right)+\sum_{j=1}^{n} a_{i j} \mathbf{h}_{j}\left(\mathbf{x}_{j}(k)\right),
$$

$$
i=1,2, \ldots, n,
$$

where $k$ represents the discrete point. $\mathbf{x}_{i}(k)=\left(x_{i 1}(k), x_{i 2}(k)\right.$, $\left.\ldots, x_{i N}(k)\right)^{T} \in \mathbf{R}^{N}$ expresses the state vector of $i$ node. $\mathbf{f}_{i}\left(\mathbf{X}_{i}(k)\right)=\left(f_{i 1}\left(\mathbf{x}_{i}(k)\right), f_{i 2}\left(\mathbf{x}_{i}(k)\right), \ldots, f_{i N}\left(\mathbf{x}_{i}(k)\right)\right)^{T} \in \mathbf{R}^{N}$ denotes the dynamic function of $i$ node.

$$
\mathbf{h}_{j}\left(\mathbf{x}_{j}(k)\right)=\left(h_{j 1}\left(\mathbf{x}_{i}(k)\right), h_{j 2}\left(\mathbf{x}_{i}(k)\right), \ldots, h_{i N}\left(\mathbf{x}_{j}(k)\right)\right)^{T} \in \mathbf{R}^{N}
$$
is the output function of $j$ node. The coupled matrix $A=$ $\left(a_{i j}\right)_{m \times n}$ illustrates the topological structures in HAPS network.

We assume that $k=k_{1}=(0,+\infty)$, and the initial network topology is changing. For discrete time complex dynamical network, there are the following three situations $[29,30]$.

Case 1. Network node has not changed. But some of the edge network weights are changed. As is shown in Figures 4(a) and 4(b), $A(1,4)$ ranges from -0.8 to -0.5 . $A(5,8)$ ranges from 0 to 0.9 . $A(6,8)$ ranges from -0.5 to 0 .

Case 2. Some nodes disappeared in the network that is associated with the removed node on the edge of weight to 0 .

As is shown in Figures 4(a) and 4(c), if node 8 is removed, $A(1,8)$ ranges from -0.8 to -0.5 . $A(8,7)$ ranges from -0.8 to 0 . $A(6,8)$ ranges from -0.5 to 0 .

Case 3. A new node is joined to the network and connected to some existing nodes. These new sides have their respective weights. At the same time in the network, the already existing edge has not changed. As can be seen from Figures 4(a) and $4(\mathrm{~d})$, node 9 is newly joined, $A(9,3)=0.9, A(9,5)=0.1$.

\section{The Principle of Traditional Channel Estimation}

3.1. Least Squares Channel Estimation Principle. Least Squares (LS) is a kind of channel estimation algorithm, where the complexity is low. When the input signal of pilot frequency $X$ and the output signal $Y$ are given, the channel estimation value $\widehat{H}_{\mathrm{ls}}$ can be obtained. The algorithm of LS channel estimation can be described as [31, 32]

$$
\widehat{H}_{\mathrm{ls}}(k)=\frac{Y(k)}{X(k)}=H(k)+\frac{\sigma(k)}{X(k)},
$$

where $\widehat{H}_{\mathrm{ls}}(k)$ is the channel estimation value of LS algorithm, $H(k)$ is the channel frequency response of LS algorithm, and $\sigma(k)$ is additive white Gaussian noise (AWGN).

Mean-Squared Error (MSE) of LS algorithm can be expressed as

$$
\mathrm{MSE}=\frac{\beta}{\mathrm{SNR}} .
$$

In the above formula, $\beta$ is the modulation constant, which is related to the channel characteristic. Signal Noise Ratio (SNR) of LS algorithm can be described as

$$
\mathrm{SNR}=\frac{E\{|x|\}^{2}}{\sigma^{2}(k)} .
$$

As can be known by formulas (1), (2), and (3), the LS algorithm does not need to know the statistical features of channel in advance. But the noise has influence on the channel estimation. It makes the BER (Bit Error Rate) and MSE bigger.

3.2. SVD-LMMS's Channel Estimation Principle. The channel estimation algorithm performance of criterion of Minimum Mean-Squared Error (MMSE) is better than the LS algorithm. The channel estimation algorithm of MMSE can be expressed as

$$
\widehat{H}_{\text {mmse }}(k)=R_{h h}\left(R_{h h}+\sigma_{n}^{2}\left(X X^{T}\right)^{-1}\right)^{-1} \widehat{H}_{\mathrm{ls}}(k),
$$

where $\widehat{H}_{\text {mmse }}(k)$ is the channel estimation value of MMSE algorithm. $R_{h h}$ is the autocorrelation response function of the 
wireless communication channel. By considering the effect of noise in MMSE algorithm criterion, it has a higher channel estimation accuracy and low BER but also will increase its complexity.

In the LMMSE (Linear Minimum Mean Square Error, LMMSE) algorithm criterion, $\left(X X^{T}\right)^{-1}$ is replaced by $E\left[\left(X X^{T}\right)^{-1}\right]$. The channel estimation algorithm of LMMSE can be expressed as $[33,34]$

$$
\begin{aligned}
\widehat{H}_{\text {lmmse }}(k) & =R_{h h}\left(R_{h h}+\sigma_{n}^{2} E\left[\left(X X^{T}\right)^{-1}\right]\right)^{-1} \widehat{H}_{\mathrm{ls}}(k) \\
& =R_{h h}\left(R_{h h}+\frac{\beta}{\mathrm{SNR}}\right)^{-1} \widehat{H}_{\mathrm{ls}}(k) .
\end{aligned}
$$

In the above formula, if the channel modulation method is QPSK, $\beta$ is equal to 1 , and if the channel modulation method is 16 QAM, $\beta$ is equal to $17 / 9$.

Due to the correlation characteristics of the HAPS channel, $\beta /$ SNR can be set to a fixed value, and the inverse matrix of $R_{h h}+\beta /$ SNR can be calculated only at one time, so it can reduce the complexity of the algorithm effectively.

But with the increase of SNR, inverse operation will be more complex. In order to further improve channel estimation accuracy of LMMSE algorithm criterion and reduce the complexity of the algorithm criterion, the channel response autocorrelation function $R_{h h}$ can be simplify processed by using the singular value decomposition (SVD) method. The SVD of $R_{h h}$ can be described as

$$
R_{h h}=U A U^{H},
$$

where $U$ is a singular vector of the unitary matrix, $A$ is a diagonal matrix singular value, and the diagonal elements are by order of singular value $\lambda_{1} \geq \lambda_{2} \geq \cdots \geq \lambda_{n} \geq 0$.

So the LMMSE channel estimation based on SVD decomposition criteria can be obtained as follows [35]:

$$
\begin{aligned}
\widehat{H}_{\text {svd-lmmse }}(k) & =U\left[A\left(A+\frac{\beta}{\mathrm{SNR}}\right)^{-1}\right] U^{H} \widehat{H}_{\mathrm{ls}}(k) \\
& =U\left[\begin{array}{cc}
\Delta_{i} & 0 \\
0 & 0
\end{array}\right] U^{H} \widehat{H}_{\mathrm{ls}}(k) .
\end{aligned}
$$

In the above formula, matrix $\Delta_{i}$ is the top left corner $i * i$ of the matrix $\Delta$, and the diagonal elements can be described as

$$
i_{k}= \begin{cases}\frac{\lambda_{k}}{\left(\lambda_{k}+\beta / \mathrm{SNR}\right)}, & k=1,2, \ldots, i \\ 0, & k=i+1, \ldots, N .\end{cases}
$$

Although, compared with traditional LMMSE algorithm, the SVD-LMMSE algorithm further simplifies the complexity of the system implementation. However, the accuracy of channel estimation can obviously do well and improve further.

\section{Proposed Channel Estimate and Modeling}

Each time-varying tap coefficient $h(n, l)$ of HAPS channel can be set up by base function $b_{q}(n)$ and base coefficient $g_{q}(l)$. Specifically, the formula is as follows:

$$
\begin{aligned}
& h(n, l)=\sum_{q=0}^{Q} b_{q}(n) g_{q}(l) \\
& \quad n=0,1, \ldots, N-1, l=0,1, \ldots, L-1,
\end{aligned}
$$

where $Q$ is the order. $Q \geq 2\left\lceil f_{d \max } N T_{s}\right\rceil f_{d \max }$ denotes the maximum Doppler frequency shift.

$T_{s}$ denotes the sampling interval. Formula (9) can be expressed in matrix form as follows:

$$
\mathbf{h}_{l}(l)=\mathbf{B g}_{l},
$$

where

$$
\begin{aligned}
\mathbf{h}_{l} & =[h(0, l), h(1, l), \ldots, h(N-1, l)], \\
\mathbf{g}_{l} & =\left[g_{0}(l), g_{1}(l), \ldots, g_{\mathrm{Q}}(l)\right]^{\mathrm{T}}, \\
\mathbf{b}_{q} & =\left[b_{q}(0), b_{q}(1), \ldots, b_{q}(N-1)\right]^{\mathrm{T}}, \\
\mathbf{B} & =\left[\mathbf{b}_{0}, \mathbf{b}_{1}, \ldots, \mathbf{b}_{\mathrm{Q}}\right] .
\end{aligned}
$$

The base function $b_{q}(n)$ can be expressed as

$$
b_{q}(n)=\exp \left(j w_{q} n\right), \quad n=0,1, \ldots, N-1 .
$$
$\mathbf{R}_{l}$ is

It can be assumed that the channel tap covariance matrix

$$
\mathbf{R}_{l}=E\left[h_{l} l h_{l}^{H}\right] .
$$

Then with the eigenvalue decomposition of formula (13), we can get

$$
\begin{aligned}
\mathbf{R}_{l} \boldsymbol{\Phi}_{l} & =\Lambda_{l} \boldsymbol{\Phi}_{l} \\
\boldsymbol{\Lambda}_{l} & =\left[\begin{array}{llll}
\lambda_{0, l} & & & \\
& \lambda_{1, l} & & \\
& & \ldots & \\
& & & \lambda_{N-1, l}
\end{array}\right] .
\end{aligned}
$$

According to the above formulas, the matrix $\mathbf{B}$ can be drawn out.

$$
\mathbf{B}=\boldsymbol{\Phi}_{l}(:, 1: Q+1) .
$$

The variance of $l$ channel tap is $\sigma_{l}^{2}$. The correlation function of tap channel can be obtained as follows:

$$
E\left\{h(n, l) h^{*}(n-m)\right\}=\sigma_{l}^{2} J_{0}\left(2 \pi m f_{d \max } T_{s}\right) .
$$

Because there are different kinds of Doppler spectrum in the actual environment, the correlation function of $l$ tap channel can be expressed as

$$
E\left\{h(n, l) h^{*}(n-m)\right\}=\sigma_{l}^{2} \frac{\sin \left(2 \pi m f_{d \max } T_{s}\right)}{\pi m} .
$$


For multiple-input-multiple-output antenna systems, the system channel capacity can be expressed as

$$
C=\log _{2}\left(\operatorname{det}\left(I_{N_{t}}+\frac{\rho}{N_{r}} H H^{H}\right)\right) \mathrm{b} / \mathrm{s} / \mathrm{Hz},
$$

where $N_{r}$ is the number of the receive antennas and $N_{t}$ is the number of the transmit antennas. $\rho$ denotes the signal-tonoise ratio of the receiving antenna. $H$ is the channel response matrix of $N_{t} \times N_{r}$.

It is assumed that the channel is an independent fading channel, and the capacity of the antenna system can be further stated as follows:

$$
C=\left[\min \left(N_{r}, N_{t}\right)\right] B \log _{2}\left(\frac{\bar{\rho}}{2}\right) \mathrm{b} / \mathrm{s} / \mathrm{Hz},
$$

where $B$ is the channel bandwidth. $\bar{\rho}$ denotes the average signal-to-noise ratio of the receiving antenna.

In the fast moving environment, the normalized Doppler frequency shift $f_{D \max }$ can be defined as

$$
f_{D \max }=\frac{v f_{c}}{c} T_{s} N
$$

where $v$ is the moving speed of the mobile platform. $f_{c}$ is the carrier frequency.

It can be used by the normalized Mean Square Error (MSE) to measure the HAPS channel estimation performance in the simulation; it can be defined as

$$
\mathrm{NMSE}=\frac{(1 / N) E\left\{|h(n, l)-\widehat{h}(n, l)|^{2}\right\}}{(1 / N) E\left\{|h(n, l)|^{2}\right\}} .
$$

\section{Simulation Analysis}

The modulation mode of HAPS wireless communication system is 16 QAM. The number of signal carrier is 256. The coding rate is $3 / 4$. The carrier frequency is $2 \mathrm{GHz}$. The relationship between the normalized Doppler frequency shift and NMSE is shown in Figure 5.

As can be seen from Figure 5, the traditional LS method has the maximum error compared with the MMSE and the proposed model method. The simulation results show that performance of the proposed channel estimation is much better than the traditional LS and SVD-LMMS method. The relationship between simulated and theoretical CDF is shown in Figure 6.

As can be seen from Figure 6, those simulation results, which are based on the real data and the proposed mathematics model, reflect the property of physical experiment to a certain extent. The values of the simulation and the calculation of proposed mathematics model are accordant basically and also have the same change tendency.

We are also interested in checking whether the obtained magnitude variations follow a Rayleigh distribution. To verify this, the sample cumulative distribution, CDF, was calculated and then compared with the theoretical CDF (Rayleigh CDF).

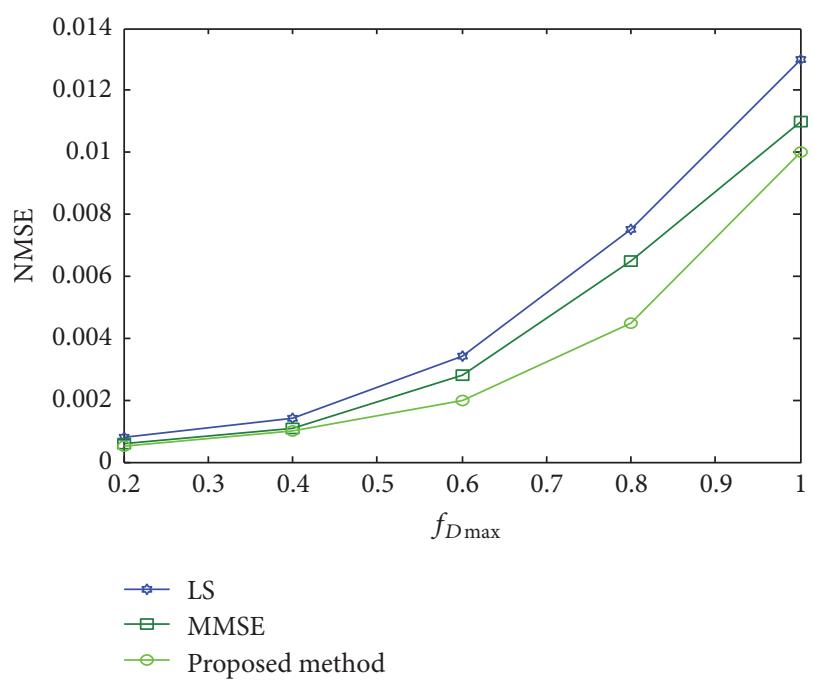

FIGURE 5: Proposed method analysis.

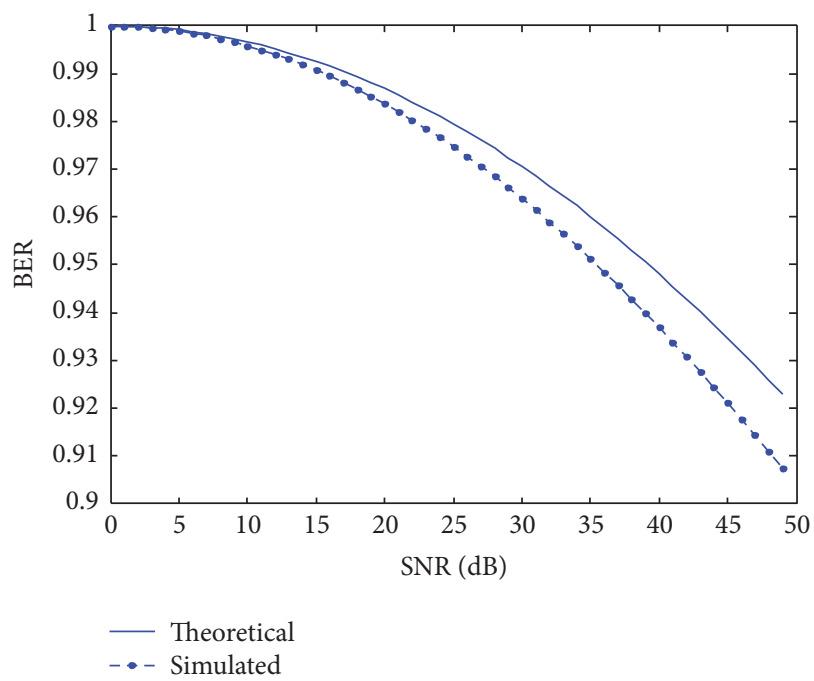

Figure 6: Proposed method analysis.

It is possible to link the simulation parameters, namely, the number of rays, $N$, and the ray amplitudes, $a_{i}$, with the Rayleigh distribution parameters; the following expression is fulfilled: $\sum_{i=1}^{N} a_{i}^{2}=N a^{2}=2 \sigma^{2}$.

As can be seen from Figure 7, it shows the simulated and theoretical CDF. A very good agreement can be observed. According to the simulation results, the proposed method and model are correct and effective. The relationship between SNR and BER is shown in Figure 8.

As can be seen from Figure 8, the new channel estimation method is superior to LS (Least Squares) channel estimation SVD-LMMS's channel estimation principle, respectively. Besides, the channel estimation performance of SVD-LMMS method is better than traditional LS method. When the BER is 0.8 , the proposed method has approximately the $3 \sim 10 \mathrm{~dB}$ system gain compared with the LS and SVD-LMMS method. 


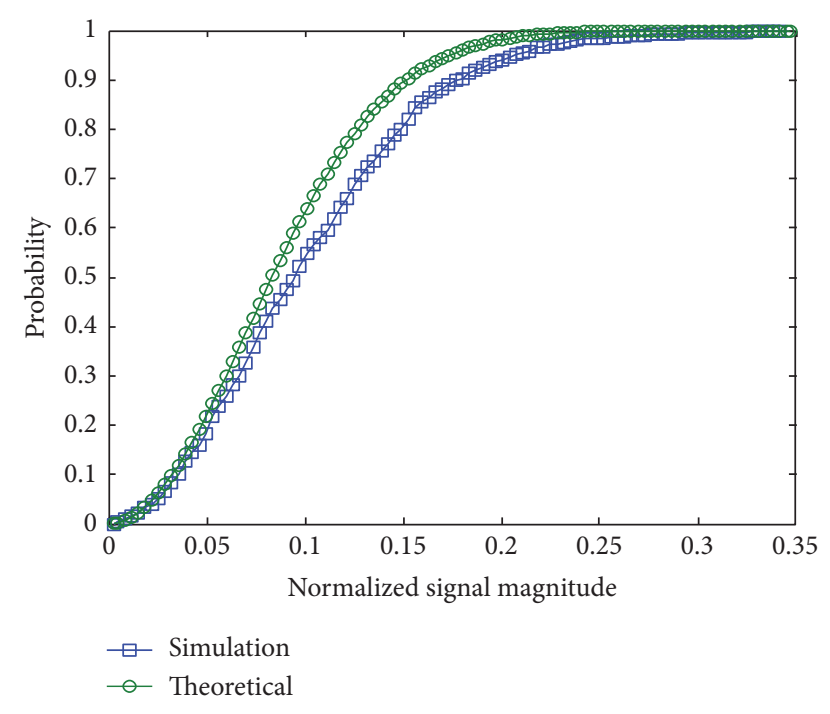

FIGURE 7: Comparison of theoretical Rayleigh and simulated CDF.

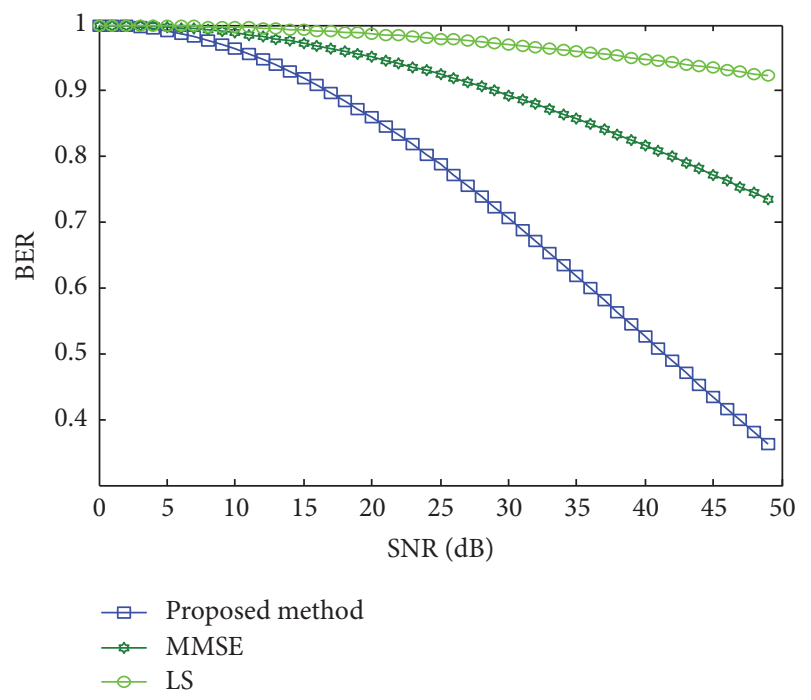

FIgURE 8: Compared with different methods.

\section{Summary}

Through the analysis of the channel characteristics of HAPS wireless communication system, some effective conclusions can be obtained by the simulation analysis. The channel characteristics of the HAPS high-speed channel fading environment are analyzed, there are two traditional channel estimation models, and methods (LS and SVD-LMMS) are introduced in the HAPS wireless communication system. In order to improve the accuracy of high-speed mobile channel estimation of HAPS systems and inhibit intercarrier interference, this paper proposes a new channel estimation algorithm and model based on basis expansion model. The simulations reveal that the performance of proposed channel estimation outperforms traditional LS and SVD-LMMS method. Result also shows that this proposed algorithm is close to the optimum at effectiveness and efficiency with much fewer computations.

\section{Conflicts of Interest}

The authors declare that there are no conflicts of interest regarding the publication of this paper.

\section{Acknowledgments}

The work was supported by Science and Technology Research Program of Chongqing Municipal Education Commission (Grants nos. KJ1600923, KJ1709206, KJ1500926, and KJ1500920), Young Research Fund Project of Humanities and Social Sciences of Ministry of Education of China (Grant no. 16YJC860010), Chongqing Social Sciences Planning Doctoral Project (2015BS059), Natural Science Foundation of China (61571069, 61501065, 61502064, 61503052, and 91438104), and Chongqing Postdoctoral Researchers Research Project Special Funded (Xm2015029).

\section{References}

[1] Z. M. Fadlullah, T. Nakajo, H. Nishiyama, Y. Owada, K. Hamaguchi, and N. Kato, "Field measurement of an implemented solar powered BS-based wireless mesh network," IEEE Wireless Communications, vol. 22, no. 3, pp. 137-143, 2015.

[2] A. Mansour, R. Mesleh, and M. Abaza, "New challenges in wireless and free space optical communications," Optics and Lasers in Engineering, 2016.

[3] D. Du, B. Qi, M. Fei, and Z. Wang, "Quantized control of distributed event-triggered networked control systems with hybrid wired-wireless networks communication constraints," Information Sciences, vol. 380, pp. 74-91, 2017.

[4] X. Liu, C. Liu, W. Liu, Y. Zhang, and X. Zeng, "HAPS wireless communication network channel modeling and simulation," in Proceedings of the 3rd International Conference on Wireless Communication and Sensor Networks (WCSN 2016), vol. 12, pp. 236-241, Wuhan, China, 2016.

[5] X. Liu, W. Liu, C. Liu, and X. Zeng, "The analysis of BER and SNR based on high altitude platform station in wireless communication network," in Proceedings of the International Conference on Communication and Electronic Information Engineering (CEIE 2016), vol. 11, pp. 17-22, Guangzhou, China, 2016.

[6] H. Elkhorchani and K. Grayaa, "Novel home energy management system using wireless communication technologies for carbon emission reduction within a smart grid," Journal of Cleaner Production, vol. 135, pp. 950-962, 2016.

[7] X. Liu, C. Liu, and W. Liu, "Wind shear target echo modeling and simulation," Discrete Dynamics in Nature and Society, vol. 2015, Article ID 479804, 2015.

[8] Liu Chao, Zheng Yang, Dihua Sun, Liu Xiaoyang, and Liu Wanping, "Stability of switched neural networks with timevarying delays," Neural Computing and Applications, no. 1, pp. 51-62, 2017.

[9] Liu Xiaoyang and Li yong, "Turbulence signal processing in the airborne weather radar," Journal of Advancements in Computing Technology, vol. 5, no. 8, pp. 816-824, 2013. 
[10] X. Ma, T. Deng, M. Xue, Z. M. Shen, and B. Lan, "Optimal dynamic pricing of mobile data plans in wireless communications," Omega, vol. 66, pp. 91-105, 2017.

[11] H. U. Yildiz, K. Bicakci, B. Tavli, H. Gultekin, and D. Incebacak, "Maximizing Wireless Sensor Network lifetime by communication/computation energy optimization of non-repudiation security service: node level versus network level strategies," Ad Hoc Networks, vol. 37, no. 1, pp. 301-323, 2016.

[12] Liu Xiaoyang, Liu Wanping, Liu Chao, and Zeng Xiaoping, "Nonlinear time-varying turbulence signal processing and simulation under curvilinear coordinate system," Communications, no. 5, pp. 1-12, 2016.

[13] W. Liu, C. Liu, and X. Liu, "A discrete dynamic model for computer worm propagation," in Difference equations, discrete dynamical systems and applications, vol. 150 of Springer Proc. Math. Stat., pp. 119-131, Springer, Cham, 2015.

[14] M. Bakula, P. Pelgrims, and R. Puers, "Wireless powering and communication for implants, based on a Royer oscillator with radio and near-field links," Sensors and Actuators A: Physical, vol. 250, no. 10, pp. 273-280, 2016.

[15] X.-P. Yang, X.-G. Zhou, and B.-Y. Cao, "Latticized linear programming subject to max-product fuzzy relation inequalities with application in wireless communication," Information Sciences, vol. 358-359, pp. 44-55, 2016.

[16] D. Jiang, Y. Wang, Y. Han, and H. Lv, "Maximum connectivitybased channel allocation algorithm in cognitive wireless networks for medical applications," Neurocomputing, vol. 220, pp. 41-51, 2017.

[17] Á. Pachón and U. M. García-Palomares, "Mid-Term frequency domain scheduler for resource allocation in wireless mobile communications systems," Computer Communications, vol. 97, pp. 96-110, 2017.

[18] N. Arianpoo and V. C. Leung, "A smart fairness mechanism for Concurrent multipath transfer in SCTP over wireless multi-hop networks," Ad Hoc Networks, vol. 55, pp. 40-49, 2017.

[19] C. Liu, W. Liu, X. Liu, C. Li, and Q. Han, "Stability of switched neural networks with time delay," Nonlinear Dynamics. An International Journal of Nonlinear Dynamics and Chaos in Engineering Systems, vol. 79, no. 3, pp. 2145-2154, 2015.

[20] X. Liu, W. Liu, C. Liu, and X. Zeng, "High Altitude platform station network and channel modeling performance analysis," Mathematics and Computer Science, vol. 7, pp. 23-31, 2016.

[21] C. Liu, W. Liu, Z. Yang, X. Liu, C. Li, and G. Zhang, "Stability of neural networks with delay and variable-time impulses," Neurocomputing, vol. 171, pp. 1644-1654, 2016.

[22] X. Liu, Y. Li, and R. Li, "The analysis of radar target echo characteristics based on radar cross section," Journal of Computational Information Systems, vol. 8, no. 18, pp. 7669-7676, 2012.

[23] X. Liu, Y. Li, and Y. Cheng, "The analysis of the side-lobe clutter in the pulse Doppler radar," Journal of Computational Information Systems, vol. 8, no. 4, pp. 1671-1677, 2012.

[24] N. McBride, J. Bulava, C. Galiotto, N. Marchetti, I. Macaluso, and L. Doyle, "Degeneracy estimation in interference models on wireless networks," Physica A: Statistical Mechanics and its Applications, vol. 469, pp. 540-550, 2017.

[25] J. Funck and C. Gühmann, "A flexible filter for synchronous angular resampling with a wireless sensor network," Measurement, vol. 98, pp. 393-406, 2017.

[26] D. G. Costa, F. Vasques, and P. Portugal, "Enhancing the availability of wireless visual sensor networks: selecting redundant nodes in networks with occlusion," Applied Mathematical
Modelling. Simulation and Computation for Engineering and Environmental Systems, vol. 42, pp. 223-243, 2017.

[27] R. Gonçalves, N. B. Carvalho, and P. Pinho, "Wireless energy transfer: Dielectric lens antennas for beam shaping in wireless power-transfer applications," Comptes Rendus Physique, vol. 18, no. 2, pp. 78-85, 2017.

[28] J. M. Portocarrero, F. C. Delicato, P. F. Pires et al., "RAMSES: a new reference architecture for self-adaptive middleware in wireless sensor networks," Ad Hoc Networks, vol. 55, no. 2, pp. 3-27, 2017.

[29] Tu. Chengyi, "Estimating the varying topology of discretetime complex dynamical networks," Central European Journal of Physics, vol. 11, no. 8, pp. 1045-1055, 2014.

[30] P. K. Sahoo and J. Sheu, "Design and analysis of collision free MAC for wireless sensor networks with or without data retransmission," Journal of Network and Computer Applications, vol. 80, pp. 10-21, 2017.

[31] W. Liu, C. Liu, Z. Yang, X. Liu, Y. Zhang, and Z. Wei, "Modeling the propagation of mobile malware on complex networks," Communications in Nonlinear Science and Numerical Simulation, vol. 37, pp. 249-264, 2016.

[32] X. Liu, "Simulation and analysis of an improved LEACH algorithm in wireless sensor network," in Proceedings of the 5th International Conference on Digital Image Processing, ICDIP 2013, vol. 8878, pp. 22-25, Beijing, China, 2013.

[33] X. Liu, C. Liu, W. Liu, and X. Zeng, "The analysis of HAPS link budget and communication performance based on near space," in Proceedings of the IEEE 11th Conference on Industrial Electronics and Applications (ICIEA), vol. 7, pp. 27-30, Hefei, China, 2016.

[34] G. Franchino and G. Buttazzo, "A power-aware MAC layer protocol for real-time communication in wireless embedded systems," Journal of Network and Computer Applications, vol. 82, pp. 21-34, 2017.

[35] F. Abayaje and P. Febvre, "A customized reduced size antipodal vivaldi antenna used in wireless baseband transmission for short-range communication," AEU - International Journal of Electronics and Communications, vol. 70, no. 12, pp. 1684-1691, 2016. 


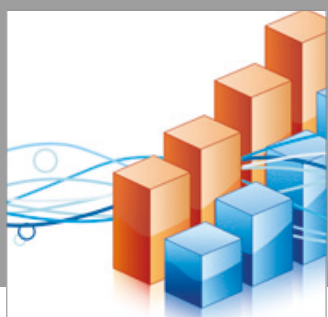

Advances in

Operations Research

vatersals

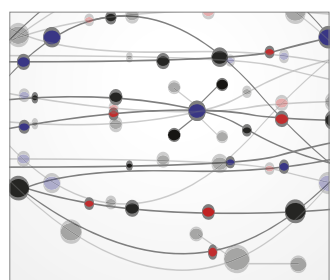

\section{The Scientific} World Journal
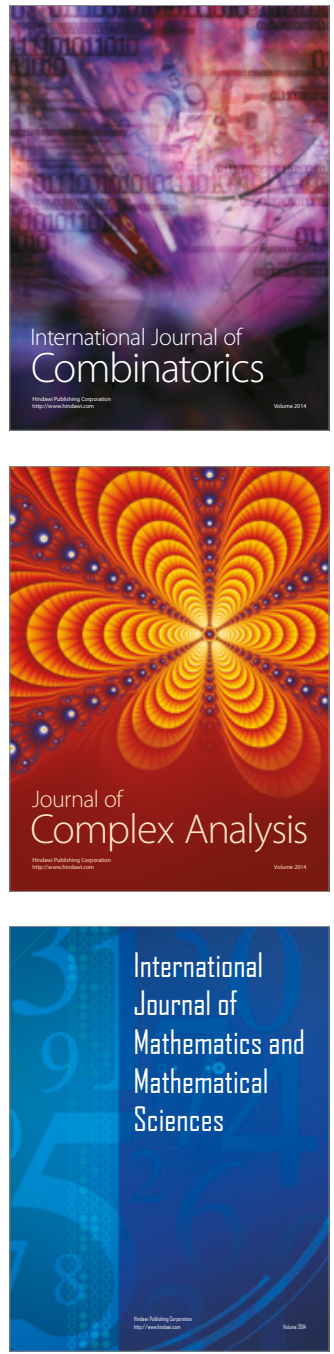
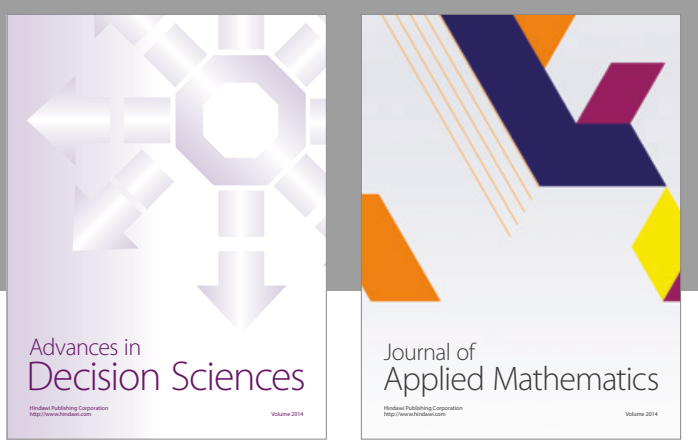

Algebra

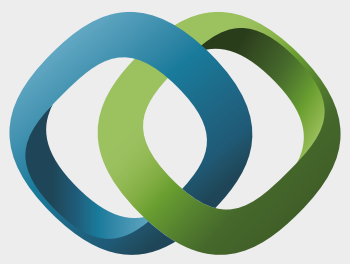

\section{Hindawi}

Submit your manuscripts at

https://www.hindawi.com
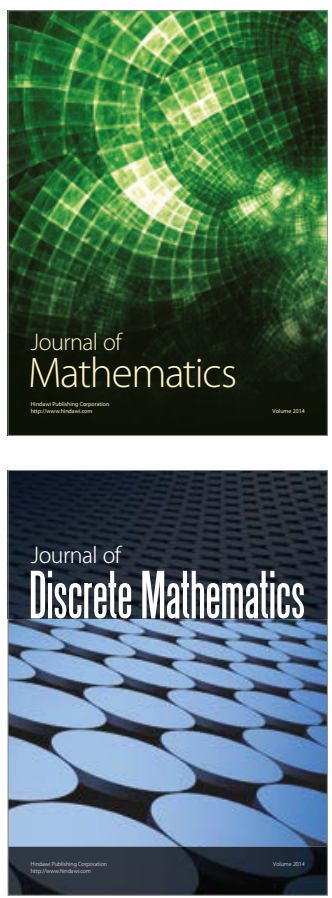

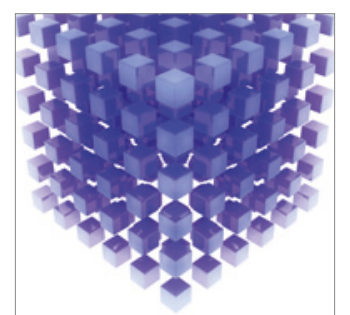

Mathematical Problems in Engineering
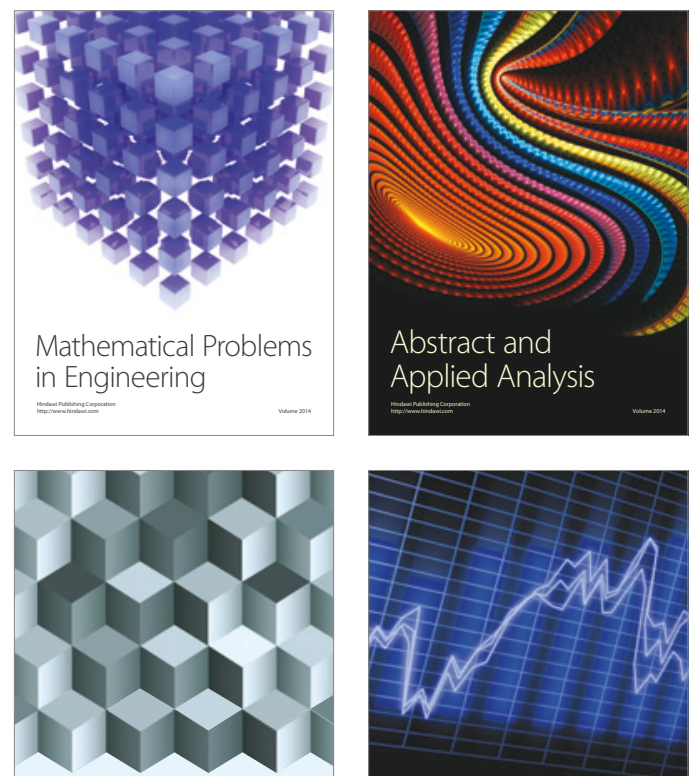

Journal of

Function Spaces

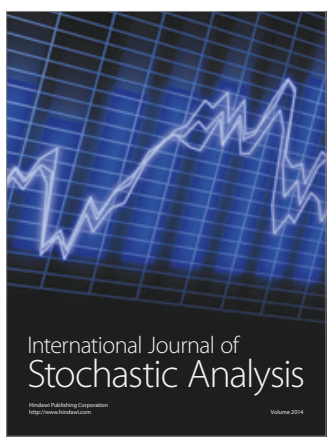

Probability and Statistics
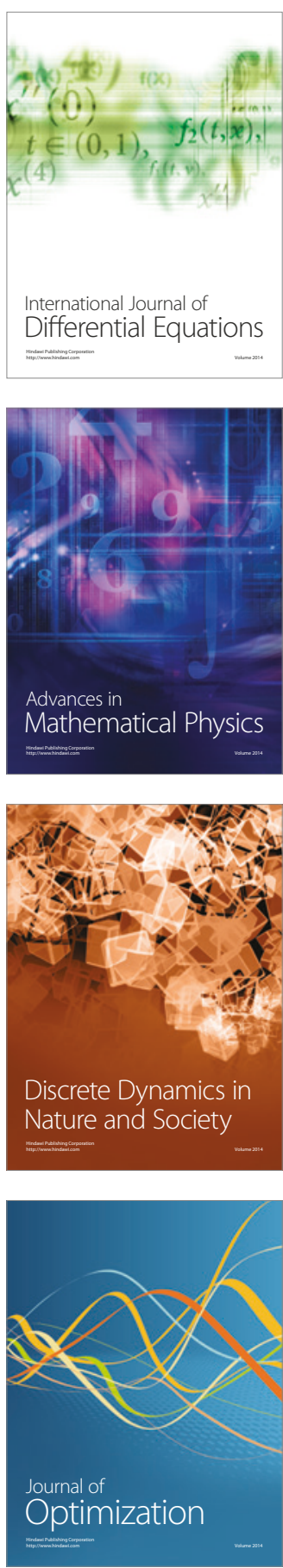